\title{
Probing Strain-Induced Phenomena in Low Dimensionality Multiferroic Oxides
}

C. Magén ${ }^{1}$, R. Guzmán ${ }^{1,2}$, S. Farokhipoor ${ }^{3,4}$, L. Maurel ${ }^{5}$, E. Langenberg ${ }^{5,6}$, J. Iñiguez ${ }^{2,7}$, S. Venkatesan ${ }^{8}$, Andrew R. Lupini ${ }^{9}$, E. Snoeck ${ }^{10}$, M. Mostovoy ${ }^{3}$, P. A. Algarabel ${ }^{6}$, J. A. Pardo ${ }^{1}$, B. Noheda ${ }^{3}$

1. Laboratorio de Microscopías Avanzadas (LMA), Instituto de Nanociencia de Aragón (INA), Univ. de Zaragoza, Zaragoza, Spain.

2. Institut de Ciència de Materials de Barcelona, ICMAB-CSIC, Barcelona, Spain.

3. Zernike Institute for Advanced Materials, Univ. of Groningen, AG Groningen, Netherlands.

4. Department of Materials Science \& Metallurgy, Univ. of Cambridge, UK.

5. Instituto de Nanociencia de Aragón (INA), Univ. de Zaragoza, Zaragoza, Spain.

6. Instituto de Ciencia de Materiales de Aragón, Univ. de Zaragoza-CSIC, Zaragoza, Spain.

7. Luxembourg Institute of Science and Technology (LIST), Esch/Alzette, Luxembourg.

8. Ludwig-Maximilians-Universität München, Munich, Germany.

9. Materials Science and Technology Division, Oak Ridge National Laboratory, Tennessee, US.

10. CEMES - CNRS, Toulouse, France.

After two decades of development, aberration-corrected STEM has established a new paradigm for nanocharacterization techniques where the chemistry, crystal and electronic structure of new materials can be determined locally, with atomic resolution and often in a quantitative way. The smart combination of STEM imaging and spectroscopy has contributed to the expansion of our understanding of the microscopic origin of physical properties [1]. The best example of the extraordinary impact of atomic resolution STEM is in the study of complex oxides. The wealth of exotic physical phenomena, exhibited upon subtle modifications of the crystal structure or composition, turns these materials into an ideal benchmark to explore the possibilities of quantitative atomically resolved STEM [2]. This lecture will focus on the application of advanced STEM techniques to the study of a specific class of oxide thin films presenting multiferroicity (i.e. the coexistence and coupling of two or more ferroic orders in a single material). The magnetoelectric coupling shown by these systems could allow them to form the core of future multifunctional devices. Bulk multiferroic materials are scarce in nature. However, in such low dimensionality systems, strategies such as strain engineering (i.e. imposing a distortion of the lattice by epitaxial strain in a controlled way) can been explored to induce or tune multiferroicity. Two examples will be reviewed here, in which the combination of STEM imaging (HAADF and ABF) and spectroscopic (EELS) techniques are key to unveil unexpected phenomena: firstly, we will review the impact of strain engineering on the magnetic properties of a well-known multiferroic material, $\mathrm{TbMnO}_{3}$. It will be demonstrated that a new two-dimensional ferromagnetic phase is synthesized at the domain walls, induced by epitaxial strain in $\mathrm{TbMnO}_{3}$ thin films [3]. Secondly, the onset of a strain-induced polar state in antiferromagnetic $\mathrm{SrMnO}_{3}$ thin films will be explored, as well as the transition from a homogeneous polar structure into a polar-graded ultrathin film as a function of thickness [4] [5].

\section{References:}

[1] "Scanning Transmission Electron Microscopy", ed. S J Pennycook and P D Nellist (Springer New York, 2011).

[2] M Varela, J Gazquez and S J Pennycook, MRS Bulletin 37 (2012) p. 29.

[3] S Farokhipoor, C Magen, S Venkatesan, J Íñiguez, C J M Daumont et al, Nature 515 (2014), p. 373.

[4] R Guzmán, L Maurel, E Langenberg, A R. Lupini, et al, Nano Letters 16 (2016), p. 2221. 
[5] Research at ORNL supported by Division of Materials Sciences and Engineering Division, Office of Basic Energy Sciences, U.S. DOE.

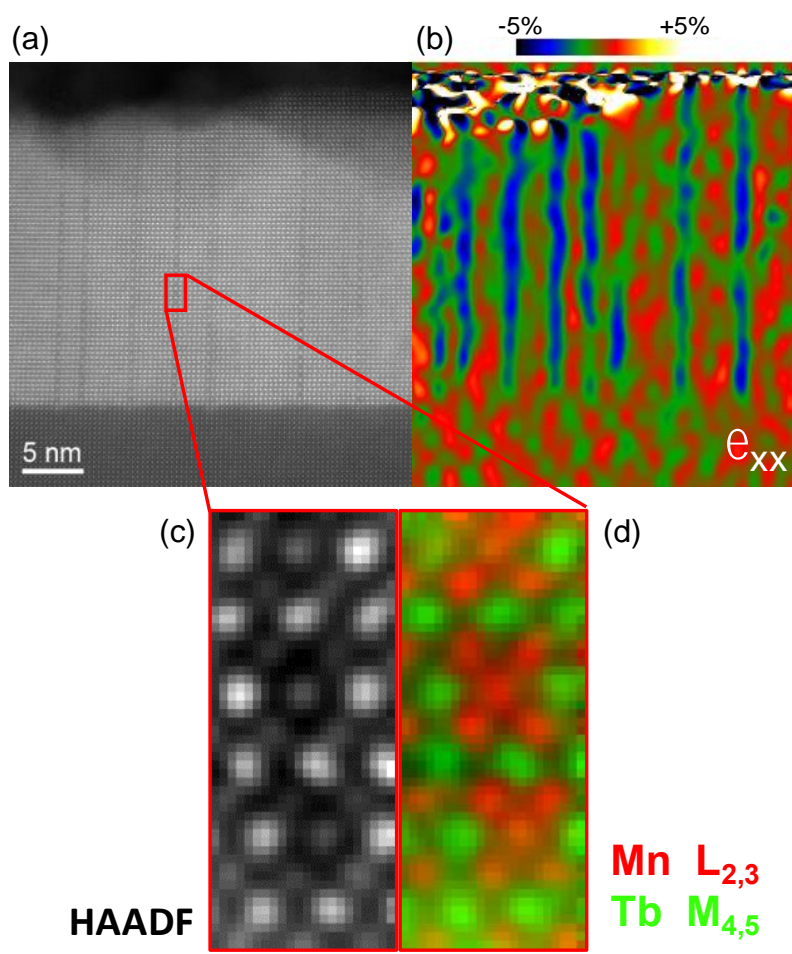

Figure 1. Ferromagnetic domain walls in strained $\mathrm{TbMnO}_{3}$ thin films. (a) HAADF-STEM image of a 25-nmthick $\mathrm{TbMnO}_{3}$ film grown on $\mathrm{SrTiO}_{3}$ (100). (b) In-plane strain map calculated by Geometrical Phase Analysis of (a). (c-d) Atomic resolution STEM-EELS spectrum image of a $\mathrm{TbMnO}_{3}$ domain wall: (c) HAADF signal, (d) color coded map of the EELS integrated intensities of the $\mathrm{Mn} \mathrm{L}_{2,3}$ and the $\mathrm{Tb} \mathrm{M}_{4,5}$ edges.

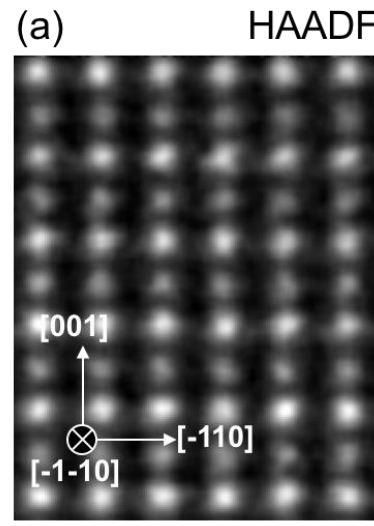

$\mathrm{SrMnO}_{3}$ (b)

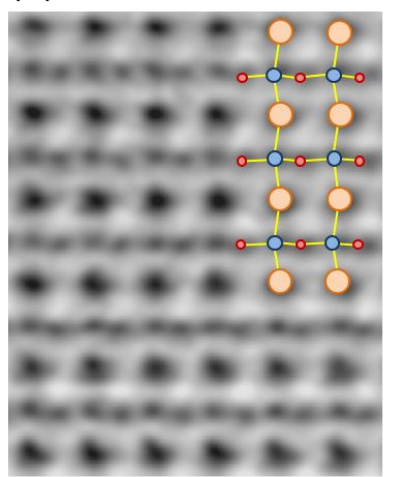

$\mathrm{Sr} \circ \mathrm{Mn} \circ \mathrm{O}$

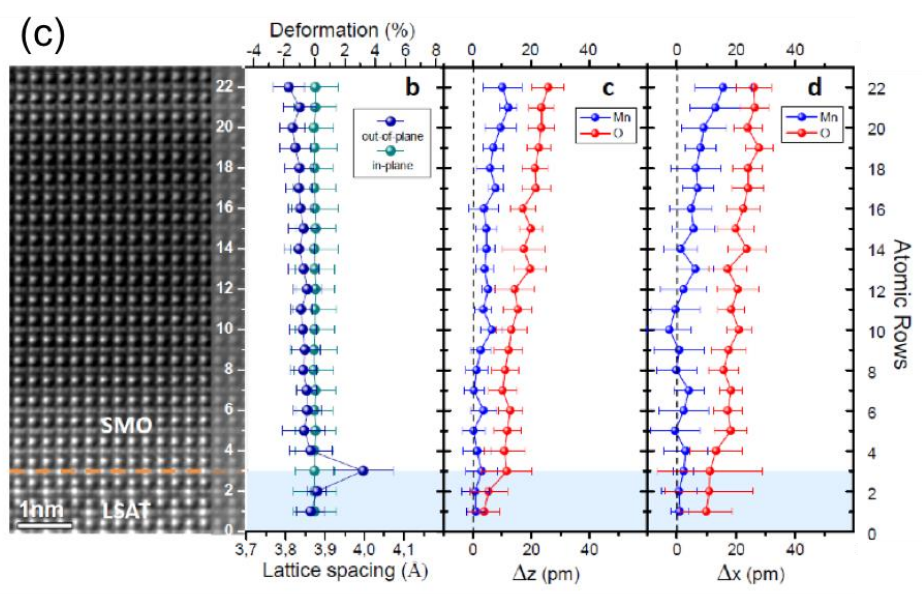

Figure 2. Polar-graded multiferroic $\mathrm{SrMnO}_{3}$ thin films. (a) HAADF-STEM image of a 10-nm-thick $\mathrm{SrMnO}_{3}$ film grown on LSAT (100). (b) ABF-STEM image of the same film, where the off-centering of the Mn and equatorial $\mathrm{O}$ atoms is illustrated with a colored atomic model. (c) Quantitative analysis of the lattice deformation and polar displacements of $\mathrm{Mn}$ and equatorial $\mathrm{O}$ in $\mathrm{SrMnO}_{3}$ thin film determined from an inverted-contrast $\mathrm{ABF}$ image. 